\title{
A Low-cost RFID/GPS Location Sensing Algorithm for Urban Infrastructure
}

\author{
http://dx.doi.org/10.3991/ijoe.v11i4.4319 \\ Changfeng Jing, Mingyi Du and Likun Shang \\ Beijing University of Civil Engineering and Architecture, Beijing, China
}

\begin{abstract}
Low-cost Radio Frequency Identification (RFID) location sensing algorithm is practical and crucial for urban infrastructure location. Current RFID location algorithms are based on fixed multiple RFID reader or reference points (reader or tag) with known position, which is cost and difficult to deployment in urban environment. The objective of this study is to develop a low-cost location sensing algorithm using RFID technique to locate urban infrastructure. This paper presents an algorithm based on multilateration with the integration of mobile RFID and Global Position System (GPS). The mobility of RFID reader can measure many distances between different RFID reader positions and target tag which represent multilateration. The GPS provides coordination of RFID reader. The location of tag is calculated based on triangle geometric property. The redundant observation is adopted to get the optimum location. In practice, the only one RFID reader is necessary, thus the cost is reduced hardly. The performance and accuracy assessment are conducted in field experiment. The experiment proves that the proposed low-cost location sensing algorithm is able to location the urban infrastructure with smaller position error. The lower cost makes it even more economically feasible than other algorithm.
\end{abstract}

Index Terms-low-cost location; RFID technique; Global Position System (GPS); urban infrastructure

\section{INTRODUCTION}

Urban infrastructure location is the crucial information for urban management and location-based services. Location data can be used for a variety of services to mobile-device users, including advertisements, billing, information, tracking and safety. According to Gartner Inc., location-awareness is one of the key technologies in the next few years [1]. Location services are traditional determined by using network and/or mobile-device-based technology [2]. Technologies supporting this include cell of origin (also known as cell ID), Angle of arrival (AOA), time of arrival (TOA) and Global Position System (GPS) or assisted GPS. GPS can be used without network modification but requires signal availability.

Automated locating technologies such as Radio Frequency Identification (RFID) have proven to be beneficial in management and tracking urban infrastructures. Kikushige used RFID to identify the roadside tree [3]. Ning introduced RFID to vehicle location [4]. Liu researched location of urban signboard by RFID [5]. Torrent established a method to automate the identification and localization of construction components [6]. However, the RFID tag coordination was jointed computed by fixed RFID reader network with known coordination $[4,7,8]$ in these studies. Thus, the network must be covered of almost study area. This is much cost and difficult to deploy because of the power source or signal blocking, especially in urban area. Many improved algorithm based on LANDMARC [9] were developed to reduce cost. Active RFID-based localization using virtual reference is developed by Zhao [10]. Huang improved the accuracy with the help of virtual landmark and topology [11]. Zhao established a kind of virtual grid to increase indoor location accuracy [12]. These researches still need set up a large number of tags as reference points in advance. The cost is still a problem.

This paper presents a low-cost algorithm based on multilateration to estimate tag location by mobile RFID reader equipped with GPS. This algorithm only needs one RFID reader with GPS module. The cost is reduced hardly. The location accuracy was analyzed from field experiment results. It was observed that the newly developed algorithm provides a high accuracy for location of urban infrastructure. The lower cost makes it even more economically feasible than other algorithm.

The purpose of this study is to develop a low-cost location sensing algorithm using RFID and GPS technologies for urban infrastructure location services. This paper is organized as follow. In section2, we take an overview of RFID location methods. Section 3 presents the newly developed algorithm. In section 4, we evaluate the performance and accuracy of the proposed algorithm. Finally, section 5 concludes the paper and gives suggestion for future work.

\section{OVERVIEW OF RFID LOCATION METHODS}

Three main methods have been developed in location sensing techniques: distance estimation, proximity techniques and scene analysis [13]. In locating of urban infrastructure, the scene analysis has not been considered as much research since the complexity of urban scene such as fading from tree and interferences.

\section{A. Distance estimation}

Trilateration and triangulation are the two techniques based on distance estimation algorithm. They use the geometric properties of triangles to compute object locations.

Trilateration uses received signal strength (RSS) or time of flight to measure the distance between emitter and receiver. Trilateration largely depends on the signal propagation nature of location environment. The error caused by multipath, fading and interferences in the environment affects the distance accuracy. The distance depends on the propagation time of the signal therefore 
having to deal with the problem of time synchronization $[13,14]$. The attenuation of the signal strength is a function of the distance between the emitter and receiver [15]. Triangulation uses angle of arrival (AOA) to estimate the target tag position by measuring the angles of at least two reference points to intersect by the two angles [16]. Multiple antennas with known separation measure the time of arrival of a signal. With the differences in propagation time and known geometric relations, the angel from the emission source is estimated. Under the principle of triangle geometry, the position of target tags can be calculated.

\section{B. Proximity technique}

A proximity location sensing technique estimates object's approximate position when it is "near" a known location or falling into limited range. The proximity technique requires the measurement of a set of neighboring reference points near by the target object, which are fixed and have known coordination [17]. Thus, the efficient proximity algorithm ensures the rapid and precise search of neighboring points. In practice, the accuracy relies on dense deployment of RFID readers to determine how near a target tag is. There are three general approaches to sensing proximity: detecting physical contact, monitoring wireless cellular access points and observing automatic ID systems [8, 18].

\section{PROPOSED ALGORITHM}

Many studies have been conducted in location sensing algorithm based on concept model with either mathematical or theoretical concepts. Ko developed algorithm for indoor tracking based on RSSI to locate the passive tags in $3 \mathrm{D}$ by static RFID readers and reference tags with known position [19]. Montaser presents a low cost indoor location identification and material tracking methodology for construction projects using Ultra High Frequency (UHF) RFID technology [20]. A number of passive RFID tags are deployed onsite as reference points with known location to determine the location of target RFID. However, these researches used a number of readers and reference tags which mean much cost for location.

In order to overcome the cost challenge, an economical outdoor location sensing algorithm is presented to estimate the location of urban infrastructure.

\section{A. Location device}

The main components of location device are GPS module and RFID reader module. The RFID reader module used to sense and locate target tags. The GPS module provides the precise position of RFID reader, which is shown in Figure 1(a). The RFID reader is shown in Figure 1(b). The tag is in Figure 1(c). In location environment, the target tags are deployed on urban infrastructure, the vehicle or worker equipped with one location device is necessary to locate. When the worker is moving with location device, the position of tag or urban infrastructure can get. In this location sensing resolution, only one RFID reader with GPS module is necessary. Thus, it is low cost than other technique. Its lower cost makes it even more economically feasible than other algorithm.

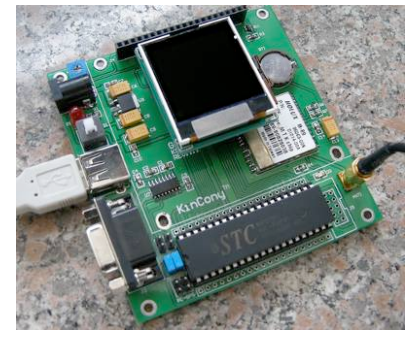

(a)

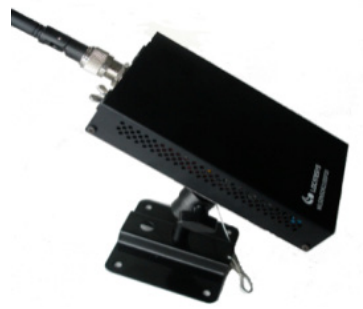

(b)

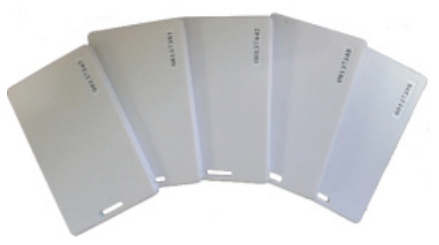

(c)

Figure 1. Pictures of GPS module (a), RFID module (b) and RFID $\operatorname{tags}(\mathrm{c})$

\section{B. The principle of location algorithm}

In traditional location methods, many RFID readers or tags composed the RFID network which is more expensive and has difficulty in device deployment in urban environment. In our research, the mobile RFID reader is employed to simulate virtual RFID readers. This is the core of our proposed algorithm.

The target object is equipped with a RFID tag which is low cost. When the target falls in the range of RFID reader, the reader captures the signal and measures the distance between reader and tag. With the moving of RFID reader, we can get many distances of different reader positions. Each distance represents one length of side. Then the target position can be calculated with multilateration.

As shown in Figure 2, the location device is moving from $\mathrm{A}$ to $\mathrm{B}$, and to $\mathrm{C}$ along the vehicle route. Then, $\mathrm{A}, \mathrm{B}$ and $C$ represent three virtual of RFID reader at different position, whose location are given by GPS.

In Figure3, the $P$ is the target point, whose position is represented by coordinate $\left(\mathrm{x}_{\mathrm{p}}, \mathrm{y}_{\mathrm{p}}\right)$. The distance between $A$ and $P$ is represented by $\mathrm{d}_{\mathrm{a}}$. The $\mathrm{d}_{\mathrm{b}}$ is distance between $B$ and $P$.

Therefore, we get two formulas as the follow:

$$
\begin{gathered}
\left(x_{p}-x_{a}\right)^{2}+\left(y_{p}-y_{a}\right)^{2}=d_{a}^{2} \\
\left(x_{p}-x_{b}\right)^{2}+\left(y_{p}-y_{b}\right)^{2}=d_{b}^{2}
\end{gathered}
$$

Where $\left(\mathrm{x}_{\mathrm{a}}, \mathrm{y}_{\mathrm{a}}\right),\left(\mathrm{x}_{\mathrm{b}}, \mathrm{y}_{\mathrm{b}}\right)$ are the coordinate of position $A$ and position $B$ respectively. The coordinate of $A$ and $B$ can be acquired from GPS module and regarded as known 
parameters. Thus, the coordinate of $P$ can be calculated through mathematics formula (1).

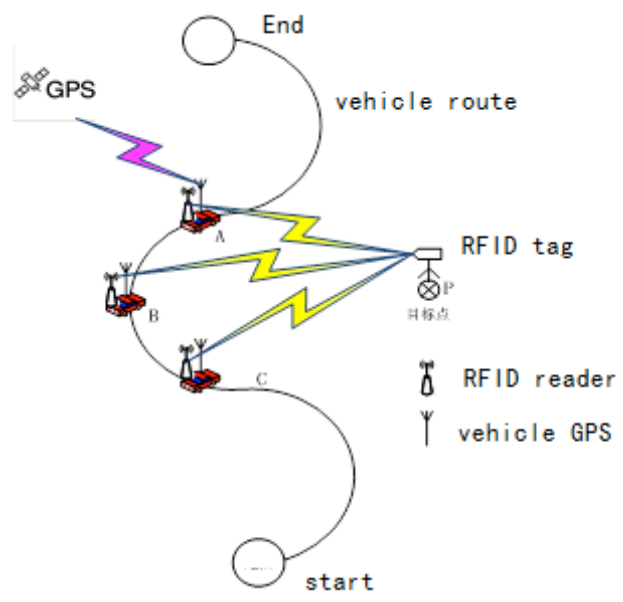

Figure 2. Principle of location

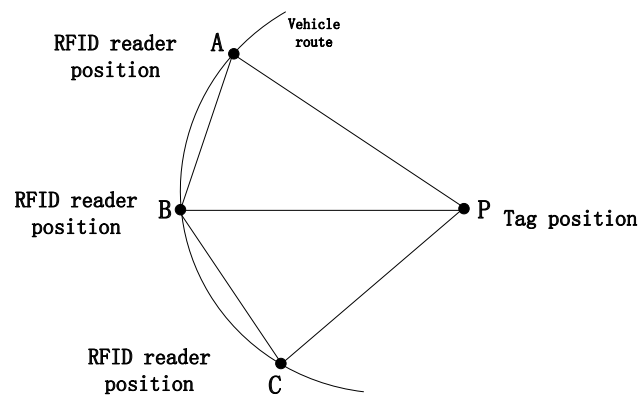

Figure 3. Graphic relation of several important points

Since the existing random error in above algorithm such as GPS error, the accuracy of location is unstable. In order to overcome this challenge, we adopted redundant observation in our research. Thus, we can get more formulas than formula (1). We used three formulas in order to reduce computation complexity. For scene shown in figure 3, we can get formulas as the follow:

$$
\begin{gathered}
\left(x_{p}-x_{a}\right)^{2}+\left(y_{p}-y_{a}\right)^{2}=d_{a}^{2} \\
\left(x_{p}-x_{b}\right)^{2}+\left(y_{p}-y_{b}\right)^{2}=d_{b}^{2} \\
\left(x_{p}-x_{c}\right)^{2}+\left(y_{p}-y_{c}\right)^{2}=d_{c}^{2}
\end{gathered}
$$

We can calculate two groups coordination of target tag $P$ with small difference from above formulas. Then, the simple mean algorithm is adopted to get the final optimum location of target tag $P$.

\section{EXPERIMENT}

An experiment is designed to validate the proposed approach with newly algorithm. To the prototype algorithm assessment and little signal interference, the choice of experiment environment and experiment time is the most important element. In our study, the $200 \mathrm{~m}$ line roads with little fading and 6:00 am with little car on road are selected for experiment in Xicheng district in Beijing. The location device is mounted on one automobile with speed below $40 \mathrm{~km} / \mathrm{h}$. The target tags are pasted on some newsstands and garbage bins distributed along roadside. The purpose of the experiment is to estimate the location of target tags and compare with the true value for assessing the accuracy.

The results of experiment may be largely influenced by the precise of Vehicle-mounted GPS. Thus, the calibration of GPS is prepared in advance. A vehicle with $40 \mathrm{~km} / \mathrm{h}$ speed is used to collect experiment data. In estimation of GPS precise, the point coordinates measured by Leica GPS are regarded as the true value. We compared the vehicle-mounted GPS data and the Leica GPS data to determine the precise. Furthermore, we take the precise factor into the calculating of target tag coordinate. In estimation of distance precise, we compare the measured value by electronic distance measuring (EDM) instrument and measured RFID distance data. This factor is taken into account in location. 20 group data with intervals of $10 \mathrm{~m}$ are collected in the selected road. The measured distances are shown in Table 1. The coordinates of tags are shown in Table 2.

From Table 1, we can see that the difference lies within the range $[-3 \mathrm{~m},+3 \mathrm{~m}]$ when the threshold distance is within $160 \mathrm{~m}$. This means the precise measurement distance of RFID reader is $160 \mathrm{~m}$.

From Table 2, we see the difference falls in $[-5 \mathrm{~m},+5$ $\mathrm{m}$ ] when the distance between RFID reader and tag falls in 160 meter. In Table 2, one big closing error is $+7.33 \mathrm{~m}$, which is caused by fading of bus in line-of-sight.

\section{CONCLUSION}

This study employed the trilateration concept to develop a low-cost RFID location sensing algorithm. The mobile RFID reader with GPS module can get many distances between reader and tag which used in multilateration location sensing algorithm. In practice, the only one RFID reader is necessary. Thus, the cost is decreased hardly. The performance of the newly algorithm is verified in field experiment. The experiment results show that the proposed location sensing algorithm is able to location the urban infrastructure with smaller position error. For the complexity of urban environment and signal attenuation, the threshold value of effective distance is validated, about $160 \mathrm{~m}$. In test, the accuracy lies within [-5 $\mathrm{m},+5 \mathrm{~m}]$ which can be adopted in urban infrastructure location.

TABLE I.

DISTANCE EXPERIMENT DATA

\begin{tabular}{|c|l|c|c|c|c|}
\hline $\boldsymbol{P D}$ & $\boldsymbol{M D}$ & Difference $(\Delta)$ & $\boldsymbol{P D}$ & MD & Difference( $\Delta$ ) \\
\hline 10 & 10.13 & +0.13 & 110 & 107.49 & -2.51 \\
\hline 20 & 19.93 & -0.07 & 120 & 119.10 & -0.80 \\
\hline 30 & 29.90 & -0.10 & 130 & 127.23 & -2.77 \\
\hline 40 & 38.89 & -1.11 & 140 & 142.07 & +2.07 \\
\hline 50 & 51.32 & +1.32 & 150 & 152.93 & +2.93 \\
\hline 60 & 60.99 & +0.99 & 160 & 160.54 & +0.54 \\
\hline 70 & 72.10 & +2.10 & 170 & 174.10 & +4.10 \\
\hline 80 & 78.73 & -1.27 & 180 & 185.58 & +5.58 \\
\hline 90 & 88.32 & -1.68 & 190 & 194.38 & -4.38 \\
\hline 100 & 102.24 & +2.24 & 200 & 203.65 & +3.65 \\
\hline
\end{tabular}

Where, PD-presetdistance; MD-measured distance 
TABLE II.

CALCULATED LOCATION DATA AND ERROR

\begin{tabular}{|c|c|c|c|c|c|}
\hline \multirow{2}{*}{ No } & \multicolumn{2}{|c|}{ Calculate value } & \multicolumn{2}{c|}{ difference } & Closing error \\
\cline { 2 - 5 } & $(\mathrm{X})$ & $(\mathrm{Y})$ & $(\Delta \mathrm{X})$ & $(\Delta \mathrm{Y})$ & \\
\hline 1 & $498,846.6$ & 307259.0 & -0.906 & 3.375 & 3.49569 \\
\hline 2 & $498,849.2$ & 307257.6 & 1.718 & 2.001 & 2.63706 \\
\hline 3 & $498,849.1$ & 307257.7 & 1.625 & 2.062 & 2.62574 \\
\hline 4 & $498,847.0$ & 307258.7 & -0.503 & 3.125 & 3.15918 \\
\hline 5 & $498,846.6$ & 307259.0 & -0.906 & 3.375 & 3.49455 \\
\hline 6 & $498,849.2$ & 307257.6 & 1.718 & 2.004 & 2.63706 \\
\hline 7 & $498,849.1$ & 307257.7 & 1.625 & 2.062 & 2.62574 \\
\hline 8 & $498,847.0$ & 307258.7 & -0.501 & 3.125 & 3.15981 \\
\hline 9 & $498,848.5$ & 307254.5 & 1.014 & -1.093 & 1.48198 \\
\hline 10 & $498,854.7$ & 307256.9 & 7.218 & 1.281 & $\mathbf{7 . 3 3 1 5 7}$ \\
\hline 11 & $498,851.6$ & 307256.2 & 4.093 & 0.562 & 4.13222 \\
\hline 12 & $498,848.9$ & 307254.8 & 1.406 & -0.812 & 1.62409 \\
\hline 13 & $498,851.3$ & 307256.2 & 3.843 & 0.593 & 4.26685 \\
\hline 14 & $498,849.7$ & 307259.2 & 2.254 & 3.625 & 4.26658 \\
\hline 15 & $498,848.2$ & 307254.5 & 0.751 & -1.061 & 1.30054 \\
\hline 16 & $498,848.7$ & 307254.8 & 1.254 & -0.781 & 1.47472 \\
\hline 17 & $498,850.1$ & 307255.3 & 2.593 & -0.312 & 2.61288 \\
\hline 18 & $498,847.8$ & 307254.3 & 0.375 & -1.281 & 1.33528 \\
\hline 19 & $498,849.1$ & 307255.0 & 1.656 & -0.625 & 1.76678 \\
\hline 20 & $498,851.4$ & 307255.4 & 3.937 & -0.156 & 3.94059 \\
\hline & & & & & \\
\hline
\end{tabular}

Although the field experiment got the good result, this research is at the beginning with algorithm and prototype test. As a result, the accuracy gotten from test is lack of practical level. Signal attenuation caused by surrounding environment was not taken into account in this study. Future study will integrate signal attenuation model to improve the accuracy of tag location. One work is normalizing the strength of signal as weight for estimation of tag location. Another work is to optimize the matching between signal points and GPS points.

\section{REFERENCES}

[1] I. Gartner. (2014/11/6). Gartner Highlights Top Consumer Mobile Applications and Services for Digital Marketing Leaders[Online]. Available: http://www.gartner .com/newsroom/id/2194115

[2] A. Schmidt-Dannert, and S. M. M. SNET, "Positioning Technologies and Mechanisms for mobile Devices", In Proc. Seminar Master Module SNET2 (TU-Berlin), 2011.

[3] Y. Kikushige, N. Yabuki, and T. Fukuda, "A Management System of Roadside Trees Using RFID and Ontology", in Computing in Civil Engineering (2011), 2011, pp. 307-314.

[4] Y. Ning, W. Zhong-qin, R. Malekian, W. Ru-chuan, and A.H. Abdullah, "Design of Accurate Vehicle Location System Using RFID", Elektronika Ir Elektrotechnika, vol. 19, no. 8, pp. 105-110, 2013. http://dx.doi.org/10.5755/j01.eee.19.8.5405

[5] Y. Liu, M. Du, C. Jing, and R. Shi, "Vehicle-mounted outdoor signboard management system combining MMS and RFID technologies", In Proc., Munich, Germany, 2012, pp. 2763-2766.

[6] D. Torrent, and C. Caldas, "Methodology for Automating the Identification and Localization of Construction Components on Industrial Projects", Journal Of Computing In Civil Engineering, vol. 23, no. 1, pp. 3-13,2009. http://dx.doi.org/10.1061/(ASCE)0 887-3801(2009)23:1(3)

[7] P. Ashok, and K.B. Marie, "A Survey of Positioning Algorithms on Mobile Devices in Location Based Services", International Journal of Advanced Research in Computer Science and Software Engineering, vol. 3, no. 6, pp. 1778-1784, 2013.
[8] J. Kuriakose, S. Joshi, R.V. Raju, and A. Kilaru, "A review on localization in wireless sensor networks", in Advances in signal processing and intelligent recognition systems Springer, 2014, pp. 599-610.

[9] N. Lionel M, L. Yunhao, Y. C. Lau, And A. P. Patil, "LANDMARC Indoor Location Sensing Using Active RFID", Wireless Networks, no. 10, pp. 701-710, 2004.

[10] Y. Zhao, Y. Liu, and L. M. Ni, "VIRE: Active RFID-based localization using virtual reference elimination", In Proc. Parallel Processing, 2007. ICPP 2007. International Conference on, 2007, pp. 56. http://dx.doi.org/10.1109/icpp.2007.84

[11] S. Huang, L. Cheng, "Topological constraints based 3D localization method of RFID tags", Application Research of Computers, no. 01, pp. 52-56, 2012.

[12] G. ZHAO, H. Liu, G. Yang, G. Song, "RFID Indoor Positioning Algorithm Based on the Space Division of Virtual Grid", Computer Systems \& Applications, no. 01, pp. 83-86, 2013.

[13] J. Hightower, and G. Borriello, "Location systems for ubiquitous computing", Computer, vol. 34, no. 8, pp. 57-66, 2001. http://dx.doi.org/10.1109/2.940014

[14] N. Bulusu, J. Heidemann, and D. Estrin, "GPS-less low-cost outdoor localization for very small devices", Personal Communications, IEEE, vol. 7, no. 5, pp. 28-34, 2000. http://dx.doi.org/10.1109/98.878533

[15] M. Bouet, and A. L. Dos Santos, "RFID tags: Positioning principles and localization techniques", In Proc. Wireless Days, 2008. WD '08. 1st IFIP, 2008, pp. 1-5.

[16] H. Cai, A. R. Andoh, X. Su, and S. Li, "A boundary condition based algorithm for locating construction site objects using RFID and GPS", Advanced Engineering Informatics, 2014. http://dx.doi.org/10.1016/j.aei.2014.07.002

[17] T. Cheng, M. Venugopal, J. Teizer, and P. A. Vela, "Performance evaluation of ultra wideband technology for construction resource location tracking in harsh environments", Automation In Construction, vol. 20, no. 8, pp. 1173-1184, 2011. http://dx.doi.org/10.1016/j.autcon.2011.05.001

[18] J. Hightowe, and G. Borriello, "A Survey and Taxonomy of Location Systems for Ubiquitous Computing", Dept. Computer Sci. and Eng., Washington Univ., seattle, Tech. Rep. UW-CSE 0108-03, 2001

[19] C. Ko, "RFID 3D location sensing algorithms", Automation In Construction, vol. 19, no. 5, pp. 588-595, 2010. http://dx.doi.org/10.1016/j.autcon.2010.02.003

[20] A. Montaser, and O. Moselhi, "RFID indoor location identification for construction projects", Automation In Construction, vol. 39, no. 0, pp. 167-179, 2014. http://dx.doi.org/10.1016/j.autcon.2013. $\underline{06.012}$

\section{AUTHORS}

Changfeng Jing is with the School of Geomatics and Urban Spatial Information of Beijing University of Civil Engineering and Architecture, Beijing, 100044, China (email: jingcf@bucea.edu.cn).

Mingyi Du is with the School of Geomatics and Urban Spatial Information of Beijing University of Civil Engineering and Architecture, Beijing, 100044, China (email:sm bucea@163.com).

Likun Shang is with the School of Geomatics and Urban Spatial Information of Beijing University of Civil Engineering and Architecture, Beijing, 100044, China (email:stu_001@126.com).

This work was supported in part by the National Key Technology $\mathrm{R}$ \& D Program of China under Grant NO. 2012BAJ14B03. Manuscript received 10 December 2014. Published as resubmitted by the authors 25 June 2015 